Xiaohu QIAN, Min HUANG, Yangyang YU, Xingwei WANG

\title{
Winner determination problem with loss-averse buyers in reverse auctions
}

(C) The Author(s) 2017. Published by Higher Education Press. This is an open access article under the CC BY license (http:// creativecommons.org/licenses/by/4.0)

\begin{abstract}
Reverse auctions have been widely adopted for purchasing goods and services. This paper considers a novel winner determination problem in a multiple-object reverse auction in which the buyer involves loss-averse behavior due to uncertain attributes. A corresponding winner determination model based on cumulative prospect theory is proposed. Due to the NP-hard characteristic, a loaded route strategy is proposed to ensure the feasibility of the model. Then, an improved ant colony algorithm that consists of a dynamic transition strategy and a Max-Min pheromone strategy is designed. Numerical experiments are conducted to illustrate the effectiveness of the proposed model and algorithm. We find that under the loaded route strategy, the improved ant colony algorithm performs better than the basic ant colony algorithm. In addition, the proposed model can effectively characterize the buyer's loss-averse behavior.
\end{abstract}

Keywords reverse auction, loss aversion, winner determination, improved ant colony algorithm

Received March 29, 2017; accepted May 21, 2017

Xiaohu QIAN

College of Information Science and Engineering, State Key Laboratory of Synthetical Automation for Process Industries, Northeastern University, Shenyang 110819, China; Research Institute of Business Analytics \& Supply Chain Management, College of Management, Shenzhen University, Shenzhen 518060, China

Min HUANG $(\bowtie)$, Yangyang YU

College of Information Science and Engineering, State Key Laboratory of Synthetical Automation for Process Industries, Northeastern University, Shenyang 110819, China

E-mail:mhuang@mail.neu.edu.cn

College of Software, Northeastern University, Shenyang 110819, China

\section{Introduction}

With the advent of Internet commerce, reverse auctions have recently become a favorable tool among buying organizations to lower procurement and transaction costs (Tunca et al., 2014, Huang et al., 2016). In general, a firstprice sealed-bid reverse auction is frequently adopted to purchase goods and services (Zhou et al., 2016, Qian et al., 2017). In such auctions, bidders are required to simultaneously place bids. The one with the lowest price wins and is paid that amount. Following the same logic, when each supplier has limited capacity, the one with a lower price has a higher chance of being selected to satisfy the buyer's demand.

Although mathematical models have been constructed to investigate the winner determination (WD) problem to optimize the buyer's expected procurement cost or expected utility, most of these models assume that the buyer involves no loss aversion behavior. However, in practical reverse auctions, due to changes of the external environment, the buyer also cares about uncertain attributes, such as the delivery time, and involves loss aversion behavior (Kahneman and Tversky, 1979). Specifically, the buyer cannot observe the exact delivery time of each potential supplier at the time of a reverse auction, but rather only has an expectation of the future delivery time. Hence, the buyer's perceived utility losses from delay delivery shall be higher than perceived utility gains from non-delay delivery. Recall that cumulative prospect theory (CPT) characterizes the fact that people are more sensitive to losses than to absolutely commensurate gains (Tversky and Kahneman, 1992), which matches the buyer's boundedly rational behavior in reverse auctions with uncertain attributes. Since loss aversion has also been detected by empirical experiments in auctions (Banerji and Gupta, 2014), incorporating CPT to the traditional WD model makes the result of the theoretical analysis closer to the practical decision-making result, making it meaningful in theory and reality. 
This paper considers a reverse auction in which one buyer solicits bids from multiple suppliers with limited capacity. In addition to the traditional price attribute, the buyer cares about the delivery time which is uncertain at the time of the reverse auction. Under such circumstances, the buyer will reflect the loss aversion attitude in the current process of WD. We aim to construct a novel WD model for the proposed problem and to provide decision support for the buyer.

Our paper contributes to the reverse auction literature by incorporating the buyer's loss-averse behavior into the classical WD when the capacity of each supplier is limited. Based on CPT, a novel WD is constructed to maximize the buyer's expected utility. To ensure the feasibility of WD, a loaded route strategy is adopted. Due to the NP-hard characteristic of the problem, to derive a satisfactory solution, we propose an improved ant colony algorithm that consists of a dynamic transition strategy and a MaxMin pheromone strategy, which is termed as an improved ant colony algorithm with loaded route strategy (IACALRS). In addition, a basic ant colony algorithm with loaded route strategy (ACA-LRS) is adopted as a benchmarking method for comparison. Numerical experiments are conducted to investigate the effectiveness of the proposed model, and to show that IACA-LRS performs better than ACA-LRS.

The organization of the paper is arranged as follows. Section 2 briefly reviews the related literature. WD models based on CPT, prospect theory (PT) and expected utility (EU) are constructed in Section 3 after the presentation of the problem description and notations. Then, in Section 4, IACA-LRS and ACA-LRS are proposed to derive effective solutions for different models. Conducting some numerical experiments, the effectiveness of the novel model and proposed algorithm is shown in Section 5. Section 6 concludes the paper with future research directions.

\section{Literature review}

Adopting reverse auctions to select the winning supplier is an important procurement activity in practice (Singh and Benyoucef, 2011). Since a novel winner determination (WD) problem that incorporates the buyer's loss-averse attitude is particularly studied, this section briefly reviews two streams of captured literature according to the situation whether decision makers involve emotional behavior or not.

For the case where decision makers involve no emotional behavior, Jackson (1976) was the first to propose the auction format for radio spectrum rights. Such an auction has been applied to the allocation of airport time slots (Rassenti et al., 1982), the transaction of financial securities (Srinivasan et al., 1998) and the transportation services procurement (Remli and Rekik, 2013). Given fixed demand, Sandholm (2002) examined a deterministic WD in multiple-object auctions where bidders have preferences for sets of objects. It showed that such WD is non-polynomial-time solvable, and designed a search algorithm solution method. To derive the optimal solution, a branch-and-cut algorithm is proposed for small-scale problems (Escudero et al., 2009). To improve the quality of procurement, the buyer may care about the reputation of potential suppliers (Rekik and Mellouli, 2012). Translating reputation into unexpected hidden cost, it formulated a generalized mathematical model which can be dealt with by CPLEX solver. When the demand is uncertain and its distribution function is given, Ma et al. (2010) constructed a two-stage stochastic programming model, and showed the effectiveness of the proposed model by conducting numerical examples with commercial branch and bound solvers. When the demand distribution is unknown, Zhang et al. (2015) proposed a tractable two-stage robust model. After constructing polyhedral uncertainty sets with a data-driven approach, it conducted numerical tests to show the effectiveness of the model by proposing a reformulation solution method. On a different line, other works have focused on reverse auction-based allocation mechanism in cloud services (Wang et al., 2013; Wang et al., 2015). A survey of this research area is available in De Vries and Vohra (2003). Reviews of supplier/vendor selection with respect to issues and approaches in supply chain are made available by De Boer et al. (2001) and Ho et al. (2010). However, these works assume that the decision makers are perfectly rational. As pointed out in Section 1, decision makers will involve boundedly rational behavior when uncertain attributes are investigated.

For the case where decision makers involve emotional behavior, Huang et al. (2016) was the first to investigate WD in reverse auctions. It proposed a PT-BOCR solution method for loss aversion buyers to address WD in a multiattribute reverse auction. The effectiveness of the proposed approach is demonstrated with numerical experiments. When bidders involve anticipated regret behavior, Qian et al. (2017) constructed a novel model based on regret theory. It analyzed the impact of such boundedly rational behavior on the buyer's expected revenue and proposed to use reserve price strategy to mitigate the adverse effect of winner regret. When bidders engage in nonequilibrium strategic thinking, Qian et al. (2016) proposed using the "Level-k decision rule" to model such bounded rationality. Managerial insights were provided for buyers to determine the winner. However, only single-object reverse auctions, in which the buyer chooses only one supplier, are considered in these works.

This paper contributes to the reverse auction literature by considering a novel WD in which buyers' loss-averse attitude is particularly examined in a multiple-object reverse auction where multiple suppliers are selected. Constructing a related model based on cumulative prospect theory, an improved ant colony algorithm with loaded 
route strategy is proposed to derive an effective solution. The effectiveness of the proposed model is illustrated with numerical experiments.

\section{Problem description and formulation}

This section presents the problem studied in our paper. Then, the notations related to the problem used in the following discussion are stated. Finally, based on CPT, PT and EU, the mathematical models are constructed for further analysis.

\subsection{Problem description}

To improve the procurement efficiency, reverse auctions are adopted to purchase goods and services by the buyer. In this paper, we mainly focus on a reverse auction activity in which one buyer faces multiple suppliers with limited capacity such that a single supplier cannot satisfy the buyer's demand. For the sake of convenience, we refer to the buyer as she and a supplier as he in the following discussion. We assume that the buyer cares about two main attributes: price and delivery (Cachon and Zhang, 2006; Tang et al., 2015). The details of the winner determination (WD) problem are shown in Fig. 1.

In the reverse auction, pre-qualified suppliers are required to submit a price-delivery combination simultaneously. A supplier with a lower price and smaller delay shall have a higher chance of being selected as a winner. Due to uncertain factors such as transportation disruptions (Hishamuddin et al., 2013), the delivery time is generally uncertain at the time of auction. This paper particularly considers a novel WD, in which the buyer involves loss aversion behavior and seeks to maximize her objective function (i.e., expected utility, PT or CPT value) according to suppliers' price-delivery combinations.

Before constructing the mathematical model, we first present the notations used throughout the paper.

\subsection{Notations}

The notations related to the problem used in the following discussion are shown below.

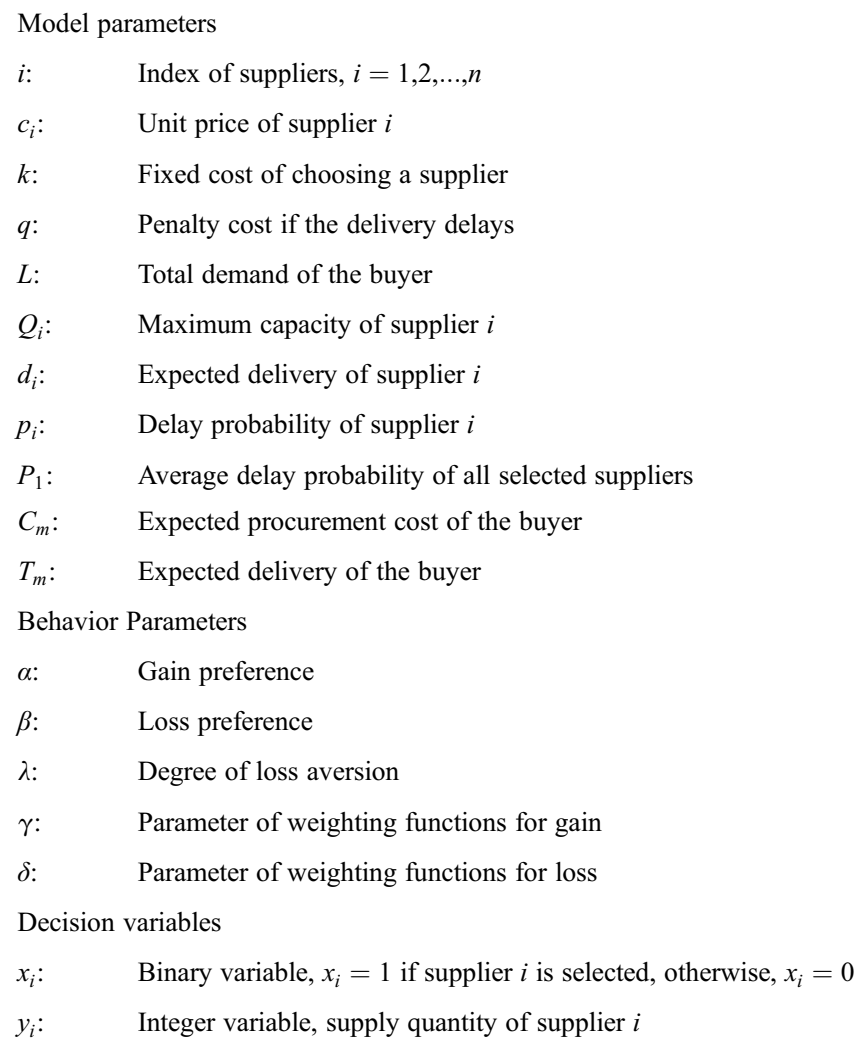

\subsection{Model formulation}

According to the above notations, the procurement cost without the delivery penalty can be characterized as

$$
C_{0}(x, y)=\sum_{i=1}^{n} c_{i} y_{i}+k \sum_{i=1}^{n} x_{i}
$$

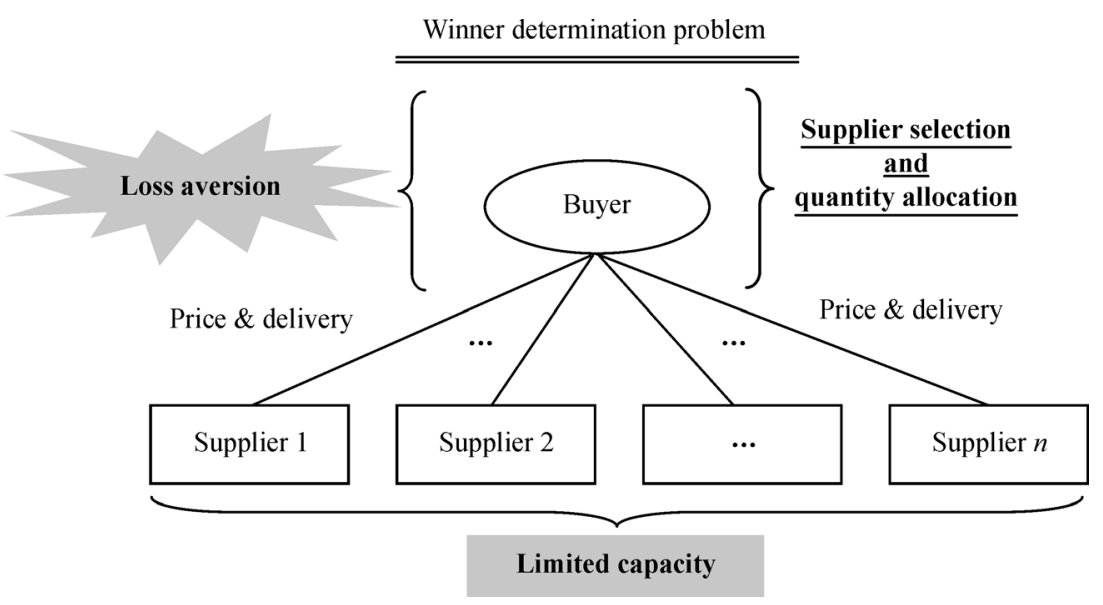

Fig. 1 Description of the winner determination problem 
where $x=\left(x_{1}, x_{2}, \ldots, x_{n}\right)$ and $y=\left(y_{1}, y_{2}, \ldots, y_{n}\right)$.

Then, the average delay probability of all selected suppliers can be expressed as

$$
P_{1}(x)=\frac{\sum_{i=1}^{n} p_{i} x_{i}}{\sum_{i=1}^{n} x_{i}}
$$

and the total delay penalty can be described as

$$
S(x)=\sum_{i=1}^{n} q\left(d_{i}-T_{m}\right)^{+} x_{i}
$$

According to the notations, the decision model based on CPT can be derived as shown below.

The total procurement cost of the buyer $C_{c}(x, y)$ can be calculated under two scenarios: i) if there is no delivery delay, then $C_{c}(x, y)=C_{0}(x, y)$; ii) if the delivery delays, then $C_{c}(x, y)=C_{0}(x, y)+S(x)$. Obviously, if $C_{c}(x, y) \leqslant$ $C_{m}$, then the buyer derives a gain of $C_{m}-C_{c}(x, y)$; otherwise, the buyer obtains a loss of $C_{c}(x, y)-C_{m}$. According to CPT, the buyer's value function can be characterized as

$$
v\left(C_{c}\right)=\left\{\begin{array}{lll}
\left(C_{m}-C_{c}\right)^{\alpha}, & \text { if } & C_{c} \leqslant C_{m} \\
-\lambda\left(C_{c}-C_{m}\right)^{\beta}, & \text { if } & C_{c}>C_{m}
\end{array}\right.
$$

Given any fixed probability $p \in[0,1]$, based on CPT, the weighting function of gain can be expressed as

$$
w^{+}(p)=\frac{p^{\gamma}}{\left(p^{\gamma}+(1-p)^{\gamma}\right)^{\frac{1}{\gamma}}}
$$

and the weighting function of loss can be expressed as

$$
w^{-}(p)=\frac{p^{\delta}}{\left(p^{\delta}+(1-p)^{\delta}\right)^{\frac{1}{\delta}}}
$$

According to the value function and the weighting function, the buyer's CPT value $V(x, y)$ can be calculated as

$$
\begin{aligned}
V(x, y)= & v\left(C_{c}(x, y)\right) \times w\left(P_{1}(x)\right)+v\left(C_{c}(x, y)\right) \times w\left(1-P_{1}(x)\right) \\
= & v\left(C_{c}(x, y)+S(x)\right) \times w\left(P_{1}(x)\right)+v\left(C_{c}(x, y)\right) \times w\left(1-P_{1}(x)\right) \\
& =\left\{\begin{array}{lll}
\left(C_{m}-C_{0}(x, y)-S(x)\right)^{\alpha} \times w^{+}\left(P_{1}(x)+C_{m}-C_{0}(x, y)\right)^{\alpha} \times w^{+}\left(1-P_{1}(x)\right), & C_{m} \geqslant C_{0}+S \\
-\lambda\left(C_{0}(x, y)+S(x)-C_{m}\right)^{\beta} \times w^{-}\left(P_{1}(x)+C_{m}-C_{0}(x, y)\right)^{\alpha} \times w^{+}\left(1-P_{1}(x)\right), & C_{0} \leqslant C_{m}<C_{0}+S \\
-\lambda\left(C_{0}(x, y)+S(x)-C_{m}\right)^{\beta} \times w^{-}\left(P_{1}(x)-\lambda\left(C_{0}(x, y)-C_{m}\right)^{\alpha} \times w^{-}\left(1-P_{1}(x)\right),\right. & C_{m}<C_{0}
\end{array}\right.
\end{aligned}
$$

To conclude, based on CPT, the novel WD problem can be formulated as

$\max V(x, y)$

s.t.

$$
\begin{gathered}
\sum_{i=1}^{n} y_{i}=L \\
(\mathrm{CPT}-\mathrm{WD}) y_{i} \leqslant x_{i} Q_{i}, \quad i=1,2, \ldots, n \\
x_{i} \in\{0,1\}, \quad i=1,2, \ldots, n \\
y_{i} \geqslant 0, \quad i=1,2, \ldots, n
\end{gathered}
$$

The buyer's objective is to maximize the CPT value as expressed by Eq. (7). Equation (8) ensures that the buyer's demand is realized. Equation (9) is the supply capacity of each supplier. Equation (10) defines the $0-1$ decision variable. Equation (11) ensures a nonnegative allocation.

Note that if $\gamma=\delta=1$, CPT-WD degenerates to a WD model based on PT denoted by PT-WD. In contrast, if $\gamma=\delta=\lambda=\alpha=\beta=1$, CPT-WD degenerates to a WD model based on the traditional EU denoted by EU-WD, in which the buyer is loss neutral. To show the effectiveness of CPT-WD which characterizes the buyer's loss aversion behavior, we use EU-WD as a benchmarking model for further analysis.

\section{An improved ant colony algorithm with loaded route strategy}

In general, an ant colony algorithm (ACA) is robust and has been widely applied to solve a number of practical problems. Since CPT-WD is a variation of the traditional knapsack problem model, CPT-WD is a NP-hard problem. Considering the characterization of our issue and model mentioned in the previous section, to avoid infeasible solutions, ACA, rather than other heuristics, such as genetic algorithm, is proposed to solve the problem. Note that the objective function of the CPT-WD model is nonlinear, we propose an improved ant colony algorithm with loaded route strategy (IACA-LRS) to address the proposed WD. Before illustrating the algorithm in detail, the parameters of algorithm are introduced below. 


$\begin{array}{ll}j: & \text { Index of ants, } j=1,2, \ldots, m \\ A: & \text { Sets of suppliers allowed to be selected } \\ p_{i}^{j}: & \text { Probability of supplier } i \text { selected by ant } j \\ \tau_{i}: & \text { Pheromone trail of supplier } i \\ \eta_{i}: & \text { Heuristic information of supplier } i \\ a: & \text { Importance parameter of pheromone trail } \\ b: & \text { Importance parameter of heuristic information } \\ k: & \text { Number of current iterations } \\ g_{i}: & \text { Number of currently selected suppliers }\end{array}$

\subsection{Loaded route strategy}

In $\mathrm{ACA}$, a route of an ant corresponds to a solution. For the proposed WD in this paper, a route shall consist of selected suppliers. To ensure the feasibility of each route, two constraints are needed. First, each supplier can be selected only once, which corresponds to Eq. (10). In addition, the sum of the capacity provided by selected suppliers should be equal to the buyer's demand, which corresponds to Eq. (8). Hence, a tabu list is adopted to ensure Eq. (10), and LRS is designed to ensure Eqs. (8)-(9). The detailed interpretations are discussed below.

Since each supplier can be selected once in each loop, to avoid returning to already-visited suppliers, a tabu list that consists of a set of selected suppliers is constructed. Note that during the route-searching process, once a supplier is selected, the minimum of the supplier's maximum capacity and the currently unsatisfied demand is calculated as the order amount. The route search ends if the minimum is no more than the maximum capacity of the selected supplier. Obviously, adopting LRS ensures that Eqs. (8), (9) and (11) are satisfied simultaneously. Therefore, we shall see that the tabu list and LRS together ensure the feasibility of the obtained route.

\subsection{Fitness function}

In general, the objective function is adopted as a fitness function to evaluate the performance of the current solution.

\subsection{Transition strategy}

According to the above parameters, a static transition probability with which ant $j$ chooses supplier $i$ in the ant colony algorithm with a loaded route strategy (ACA-LRS) can be expressed as

$$
p_{i}^{j}=\frac{\tau_{i}^{a} \eta_{i}^{b}}{\sum_{k \in A} \tau_{i}^{a} \eta_{i}^{b}}
$$

$\begin{array}{cl}\rho: & \text { Pheromone evaporation rate, } \rho \in[0,1] \\ \tau_{\max }: & \text { Maximum pheromone trail } \\ \tau_{\min }: & \text { Minimum pheromone trail } \\ Q: & \text { Total amount of pheromone presented on potential suppliers } \\ t & \text { Index of loops } \\ h_{j}^{t}: & \text { Number of selected suppliers for loop } t \text { by ant } j \\ f & \text { Initial amount of pheromone } \\ N P: & \text { Size of population } \\ N G: & \text { Number of total loops }\end{array}$

whereas the dynamic transition probability in IACA-LRS can be expressed as

$$
\begin{gathered}
p_{i}^{j}=\frac{\tau_{i}^{a} \eta_{i}^{b} \chi_{i}}{\sum_{k \in A} \tau_{i}^{a} \eta_{i}^{b} \chi_{i}} \\
\chi_{i}=\frac{m k}{m k+g_{i} \cdot \eta_{i} / \max \eta}
\end{gathered}
$$

where $\eta_{i}=1 / E_{i}, E_{i}=c_{i}\left(1-p_{i}\right)+\left(c_{i}+q_{i}\left[d_{i}-T_{m}\right]^{+} / y_{i}\right) p_{i}$ denotes the expected unit fee of supplier $i,[x]^{+}=$ $\max \{0, x\}$, and $\max \eta$ denotes the maximum amount of pheromone. Based on Eq. (13), we rearrange the probabilities such that $\hat{p}_{1}^{j} \geqslant \hat{p}_{2}^{j} \geqslant \cdots \geqslant \hat{p}_{n}^{j}$. For a randomly generated number $p \in(0,1)$, if $0 \leqslant p<\hat{p}_{1}^{j}$, then the supplier with probability $\hat{p}_{k}^{j}$ is selected; otherwise, if $\sum_{i=2}^{k} \hat{p}_{i-1}^{j} \leqslant$ $p<\sum_{i=2}^{k} \hat{p}_{i}^{j}$, then the supplier with probability $\hat{p}_{k}^{j}$ is selected.

\subsection{Pheromone update rule}

After constructing each tour to form solutions for the WD problem such that the buyer's requirement can be satisfied by the selected suppliers, the pheromone levels are updated based on the following formulas:

$$
\tau_{i}(\mathrm{t}+1)=\rho \tau_{i}(\mathrm{t})+\Delta \tau_{i}(\mathrm{t})
$$

$$
\Delta \tau_{i}(\mathrm{t})=\sum_{j=1}^{m} \Delta \tau_{i}^{j}(\mathrm{t})
$$

$$
\Delta \tau_{i}^{j}(t)= \begin{cases}Q / h_{j}^{t}, & \text { if supplier } i \text { is selected } \\ 0, & \text { otherwise }\end{cases}
$$

To avoid local maximum and improve the quality of solutions, the Max-Min pheromone strategy is adopted. Specifically, the pheromone values are limited to the interval $\left[\tau_{\min }, \tau_{\max }\right]$. Hence, we have 


$$
\begin{aligned}
& \tau_{i}(t+1) \\
= & \begin{cases}\tau_{\min }, & \text { if } \tau_{i}(t+1) \leqslant \tau_{\min } \\
\rho \tau_{i}(t)+\Delta \tau_{i}(t), & \text { if } \tau_{\min }<\tau_{i}(t+1) \leqslant \tau_{\max } \\
\tau_{\max }, & \text { if } \tau_{i}(t+1)>\tau_{\max }\end{cases}
\end{aligned}
$$

\subsection{Supply quantity determination}

Note that the buyer's objective is to select multiple suppliers such that the demand is satisfied and the expected utility is maximized. In addition, if a supplier is selected, the corresponding pheromone will increase. Hence, when a supplier is selected by an ant to form the feasible solution, the supplier will provide the maximum supply capacity if the current total supply is less than the demand, or part of the maximum supply capacity to make sure the total supply equals to the demand. In such a way, the ant forms a tour that provides a feasible solution for the buyer.

\subsection{Termination rule}

The algorithm stops and outputs the best solution after a constant loop $N G$.

\subsection{Framework of the proposed IACA-LRS}

In summary, the framework of the proposed IACA-LRS can be itemized below.

Step 1: Initialization of the IACA-LRS parameters, which consist of population size $N P$, maximum loops $N G$, current iterations $k$, initial pheromone $f$, and the current index of ant $r$.

Step 2: In each loop, to construct its tour to form solutions, each ant randomly chooses a supplier to start the algorithm, and then the selected supplier is included in the tabu list.

Step 3: Each ant chooses the next supplier using Eqs. (13)-(14), and the tabu list is updated accordingly.

Step 4: Check whether the buyer's demand $L$ is satisfied. If not, go to Step 3; otherwise, go to Step 5.

Step 5: Empty the tabu list, and update the currently best solution.

Step 6: The current ant finishes constructing its tour, and the next ant begins its tour. Let $r=r+1$.

Step 7: Check whether the number of ants is equal to the population size $N P$. If not, go to Step 2; otherwise, go to Step 8.

Step 8: Update the pheromone levels according to Eqs. (15)-(18).

Step 9: Let $k=k+1$.

Step 10: Check whether the current loop is equal to the maximum loop. If not, go to Step 2; otherwise, output the best solution and stop the process.

Note that if we replace Eq. (13)-(14) to Eq. (12) in Step
2 and Eqs. (15)-(18) to Eqs. (15)-(17) in Step 8, IACALRS becomes ACA-LRS. In addition, to check the performance of solutions derived by IACA-LRS, the enumeration method (EM) is used.

\section{Numerical experiments}

In this section, to evaluate the quality of solutions, three different examples, i.e., a small-scale example with 10 suppliers, a medium-scale example with 20 suppliers and a large-scale example with 30 suppliers, are illustrated to show the effectiveness of the proposed IACA-LRS. In the following discussion, the worst solution (WS), best solution (BS), average solution (Mean), standard deviation (SD), and average time taken by the algorithm (Time) are adopted to investigate the performance of the proposed algorithm. Specifically, after deriving optimal parameter combinations for different examples, we run these two ant colony algorithms 50 times to obtain WS, BS, Mean, SD and Time, and show that IACA-LRS performs best. Then, by fixing $C_{m}$ and varying $T_{m}$ or by fixing $T_{m}$ and varying $C_{m}$, we analyze the impact of expected delivery or expected procurement cost on the optimal solution. Finally, we compare the results of different models to show that CPT can effectively characterize the buyer's loss aversion behavior.

\subsection{Comparison analysis of different algorithms}

For different scale examples, given $C_{m}=44500, T_{m}=10$, $\alpha=\beta=0.88$, and $\lambda=2.25$, changing one of the parameters of IACA-LRS (or ACA-LRS) with all other parameters being fixed, we can derive the optimal combination of the parameters of IACA-LRS (or ACALRS) and the detailed results of the problem using different algorithms (EM, ACA-LRS or IACA-LRS) as shown in Table 1.

Table 1 shows that given the optimal combination of the parameters for different algorithms, IACA-LRS performs better than ACA-LRS and EM under different scale problems. Specifically, IACA-LRS can obtain the optimal solution for the small-scale problem, and can find better solutions than ACA-LRS in terms of medium- and largescale problems. In addition, IACA-LRS can find highquality solutions in shorter time compared to the other two algorithms.

\subsection{Analysis of effects of expected delivery}

For the small-scale problem, let $C_{m}=44500$, when $T_{m}$ varies, the different solutions are shown in Table 2.

From Table 2, we see that when the expected delivery is small, the optimal objective value is negative, which means that the current solution cannot satisfy the buyer's demand. In such cases, both the unit price and delivery 
Table 1 Results obtained with different algorithms under different scales

\begin{tabular}{|c|c|c|c|c|c|c|c|c|c|c|c|c|c|c|c|}
\hline$\overline{N^{\text {a) }}}$ & Alg ${ }^{b)}$ & $N P$ & $N G$ & $a$ & $b$ & $\bar{\rho}$ & $f$ & $Q$ & $\overline{\tau_{\min }}$ & $\tau_{\max }$ & WS & BS & Mean & SD & Time \\
\hline \multirow[t]{3}{*}{$\overline{10}$} & EM & $-^{c)}$ & - & - & - & - & - & - & - & - & 80.94 & 80.94 & 80.94 & - & 7092.64 \\
\hline & ACA-LRS & 70 & 1000 & 1.0 & 0.5 & 0.6 & 2.5 & 13 & 0.4 & 3.2 & 77.61 & 80.94 & 78.83 & 1.19 & 1.95 \\
\hline & IACA-LRS & 50 & 300 & 0.9 & 0.5 & 0.5 & 2.5 & 15 & 0.5 & 3.8 & 80.94 & 80.94 & 80.94 & 0 & 0.42 \\
\hline \multirow[t]{3}{*}{20} & EM & - & - & - & - & - & - & - & - & - & - & - & - & - & - \\
\hline & ACA-LRS & 85 & 1700 & 1.5 & 1.3 & 0.6 & 4.2 & 15 & 0.5 & 4.5 & 142.79 & 146.25 & 144.62 & 3.24 & 9.65 \\
\hline & IACA & 70 & 1500 & 1.5 & 1.2 & 0.7 & 4.0 & 17 & 0.6 & 5 & 145.78 & 149.40 & 147.19 & 1.73 & 6.42 \\
\hline \multirow[t]{3}{*}{30} & EM & - & - & - & - & - & - & - & - & - & - & - & - & - & - \\
\hline & ACA-LRS & 95 & 2000 & 1.1 & 1.2 & 0.6 & 4.0 & 17 & 0.4 & 4.5 & 120.74 & 131.05 & 125.66 & 5.29 & 26.70 \\
\hline & IACA-LRS & 80 & 1500 & 1.3 & 1.2 & 0.5 & 4.3 & 17 & 0.4 & 5 & 129.58 & 131.05 & 130.24 & 3.28 & 20.16 \\
\hline
\end{tabular}

Note: a) " $N$ " denotes the number of suppliers; b) "Alg” denotes different algorithm; c) "-" denotes non-available value.

Table 2 Effects of expected delivery on the optimal solutions

\begin{tabular}{lcccccccc}
\hline$T_{m}$ & $C_{m}$ & $C_{0}$ & $S$ & BS & WS & Mean & SD & Time \\
\hline 6 & 44500 & 44553.0 & 3000 & -309.94 & -309.94 & -309.94 & 0 & 0.42 \\
7 & 44500 & 44514.0 & 2500 & -259.06 & -259.06 & -259.06 & 0 & 0.42 \\
8 & 44500 & 44514.0 & 1900 & -204.06 & -204.06 & -204.06 & 0 & 0.42 \\
9 & 44500 & 44208.0 & 1600 & -69.81 & -69.81 & -69.81 & 0 & 0.42 \\
10 & 44500 & 44282.5 & 1000 & 80.94 & 80.94 & 80.94 & 0 & 0.42 \\
11 & 44500 & 44208.0 & 600 & 181.44 & 181.44 & 181.44 & 0 & 0.42 \\
12 & 44500 & 44208.0 & 300 & 256.81 & 256.81 & 256.81 & 0 & 0.42 \\
13 & 44500 & 44208.0 & 100 & 280.33 & 280.33 & 280.33 & 0 & 0.42 \\
14 & 44500 & 44208.0 & 0 & 291.5 & 291.5 & 291.5 & 0 & 0.42 \\
15 & 44500 & 44208.0 & 0 & 291.5 & 291.5 & 291.5 & 0 \\
\hline
\end{tabular}

penalty impact the optimal solutions, and the delivery time is more important to the buyer. When the expected delivery is medium, the importance of the unit price and delivery penalty becomes blurred. When the expected delivery is large, the unit price becomes more important to the buyer. The effects of expected delivery on the optimal solutions remain the same for medium- and large-scale problems.

\subsection{Analysis of effects of expected procurement cost}

For the small-scale problem, given $T_{m}=10$, when $C_{m}$ varies, the different solutions are shown in Table 3.

From Table 3, we see that when the expected procurement cost is small, the optimal objective value is negative. When $C_{m}$ increases, the optimal objective value becomes positive, and the buyer prefers suppliers with small unit price. The effects of the expected procurement cost on the optimal solutions remain the same for mediumand large-scale problems.

\subsection{Comparison analysis of different models}

For different scale examples, the detailed results using different models are shown in Table 4.
Note that the decision behavior of the buyer may be different. By assuming that the buyer is perfectly rational, boundedly rational with loss aversion or boundedly rational with risky prospects, respectively, we have constructed three different models, i.e., EU-WD, PT-WD or CPT-WD, with different objective functions. From Table 4, we see that the best solutions for different models are different.

Furthermore, for a small-scale problem, given $T_{m}=10$, as $C_{m}$ varies, to show the effectiveness of CPT-WD, the objective values using different models under IACA-LRS are shown in Fig. 2.

From Fig. 2, we see that compared with EU-WD, the CPT-WD can effectively characterize the buyer's lossaverse behavior such that the buyer is more sensitive to losses compared to gains. The results remain the same for medium- and large-scale problems.

\section{Conclusions}

Reverse auctions are a favorable procurement tool for buying organizations to purchase goods and services, and have become a hot research topic in recent days. To 
Table 3 Effects of expected procurement cost on the optimal solutions

\begin{tabular}{lcccccccc}
\hline$C_{m}$ & $T_{m}$ & $C_{0}$ & $S$ & BS & WS & Mean & SD & Time \\
\hline 44000 & 10 & 44208.5 & 1000 & -551.69 & -551.69 & -551.69 & 0 & 0.42 \\
44100 & 10 & 44208.5 & 1000 & -359.65 & -359.65 & -359.65 & 0 & 0.42 \\
44200 & 10 & 44208.5 & 1000 & -186.74 & -186.74 & -186.74 & 0 & 0.42 \\
44300 & 10 & 44208.5 & 1000 & -129.61 & -129.61 & -129.61 & 0 & 0.42 \\
44400 & 10 & 44208.5 & 1000 & -33.02 & -33.02 & -33.02 & 0 & 0.42 \\
44500 & 10 & 44208.5 & 1000 & 80.95 & 80.95 & 80.95 & 0 & 0.42 \\
44600 & 10 & 44208.5 & 1000 & 194.90 & 194.90 & 194.90 & 0 & 0.42 \\
44700 & 10 & 44208.5 & 1000 & 308.86 & 308.86 & 308.86 & 0 & 0.42 \\
44800 & 10 & 44208.5 & 1000 & 422.81 & 422.81 & 422.81 & 0 & 0.42 \\
44900 & 10 & 44208.5 & 600 & 536.77 & 536.77 & 536.77 & 0
\end{tabular}

Table 4 Results obtained with different models under different scales

\begin{tabular}{|c|c|c|c|c|c|c|c|}
\hline$N^{\text {a) }}$ & Model & Solutions & BS & WS & Mean & $\mathrm{SD}$ & Time \\
\hline \multirow[t]{3}{*}{10} & EU-WD & $(1,125),(2,160),(7,69),(9,55),(10,91)$ & 179.83 & 179.83 & 179.83 & 0 & 0.42 \\
\hline & PT-WD & $(1,125),(2,160),(3,110),(5,13),(10,92)$ & 50.07 & 50.07 & 50.07 & 0 & 0.42 \\
\hline & CPT-WD & $(1,125),(2,160),(7,69),(9,55),(10,91)$ & 80.94 & 80.94 & 80.94 & 0 & 0.42 \\
\hline \multirow[t]{3}{*}{20} & EU-WD & $(2,120),(4,51),(6,136),(11,64),(12,135),(14,94)$ & 291.71 & 286.37 & 288.41 & 2.14 & 6.42 \\
\hline & PT-WD & $(2,120),(4,51),(6,136),(11,64),(12,135),(14,94)$ & 76.88 & 72.37 & 74.67 & 1.89 & 6.41 \\
\hline & CPT-WD & $(2,120),(6,59),(11,64),(12,135),(14,94),(20,128)$ & 149.40 & 145.78 & 147.19 & 1.73 & 6.42 \\
\hline \multirow[t]{3}{*}{30} & EU-WD & $(11,140),(19,95),(20,86),(26,190),(30,139)$ & 170.80 & 162.58 & 168.44 & 3.59 & 20.16 \\
\hline & PT-WD & $(11,140),(19,95),(20,60),(26,190),(30,165)$ & 70.35 & 65.49 & 67.23 & 3.46 & 20.17 \\
\hline & CPT-WD & $(11,140),(19,95),(20,86),(26,190),(30,139)$ & 131.05 & 129.58 & 130.24 & 3.29 & 20.16 \\
\hline
\end{tabular}

Note: a) " $N$ " denotes the number of suppliers.

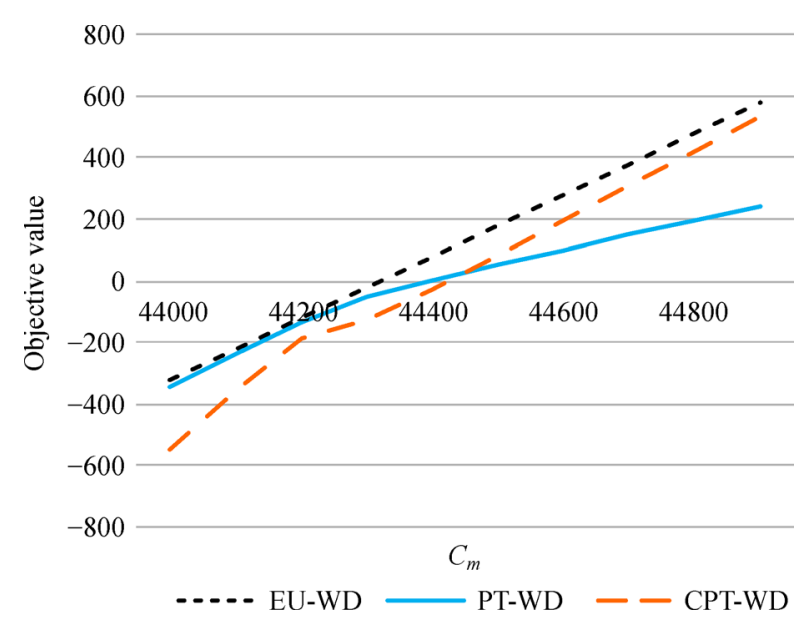

Fig. 2 Comparison analysis of different models under IACA-LRS

improve procurement efficiency, buyers also care about non-price attributes in multiple-object reverse auctions. Considering the fact that external environment may change, uncertain attributes such as delivery time are particularly considered. This paper focuses on two attributes, i.e., price and delivery time, and aims to investigate a novel winner determination problem in which the buyer involves loss-averse behavior. Assuming each supplier has limited capacity, based on the cumulative prospect theory, a nonlinear model is constructed. Specifically, the buyer's objective is to maximize the expected utility constraint on the demand and supplier's capacity. By setting the parameters in the proposed model, we can obtain winner determination models based on prospect theory and expected utility theory, respectively. To solve the problem, under a loaded route strategy, an improved ant colony algorithm that consists of a dynamic transition strategy and a Max-Min pheromone strategy is proposed. Numerical experiments show the effectiveness of the proposed algorithm by comparing to the basic ant colony algorithm with a loaded route strategy. In addition, we find that the proposed model can effectively characterize the loss-averse behavior of the buyer.

Several other interesting issues may be investigated in the future. First, the buyer may involve other boundedly rational behavior such as regret, cognitive hierarchy, and fairness concerns. Incorporating such behaviors into the traditional winner determination model is important for practical applications and theoretical studies. Second, the buyer may care about other uncertain attributes, such as the 
supply disruption, quality risk or capacity uncertainty. Investigating the winner determination problem in such settings by assuming that the buyer is loss-averse is meaningful.

Acknowledgements This work has been sponsored by the Distinguished Young Scholars Award of NSFC Grant \#71325002; the Major International Joint Research Project of NSFC Grant \#71620107003; the Foundation for Innovative Research Groups of NSFC Grant \#61621004; 111 Project Grant \#B16009; and the Fundamental Research Funds for State Key Laboratory of Synthetical Automation for Process Industries Grant \#2013ZCX11.

\section{References}

Banerji A, Gupta N (2014). Detection, identification, and estimation of loss aversion: Evidence from an auction experiment. American Economic Journal: Microeconomics, 6(1): 91-133

Cachon G P, Zhang F (2006). Procuring fast delivery: Sole sourcing with information asymmetry. Management Science, 52(6): 881-896

De Boer L, Labro E, Morlacchi P (2001). A review of methods supporting supplier selection. European Journal of Purchasing \& Supply Management, 7(2): 75-89

De Vries S, Vohra R V (2003). Combinatorial auctions: A survey. INFORMS Journal on Computing, 15(3): 284-309

Escudero L F, Landete M, Marín A (2009). A branch-and-cut algorithm for the winner determination problem. Decision Support Systems, 46 (3): 649-659

Hishamuddin H, Sarker R A, Essam D (2013). A recovery model for a two-echelon serial supply chain with consideration of transportation disruption. Computers \& Industrial Engineering, 64(2): 552-561

Ho W, Xu X, Dey P K (2010). Multi-criteria decision making approaches for supplier evaluation and selection: A literature review. European Journal of Operational Research, 202(1): 16-24

Huang M, Qian X, Fang S C, Wang X (2016). Winner determination for risk aversion buyers in multi-attribute reverse auction. Omega, 59: 184-200

Jackson C (1976). Technology for spectrum markets. Ph.D. thesis, Department of Electrical Engineering, Massachusetts Institute of Technology, Cambridge, MA

Kahneman D, Tversky A (1979). Prospect theory: An analysis of decision under risk. Econometrica, 47(2): 263-291

Ma Z, Kwon R H, Lee C G (2010). A stochastic programming winner determination model for truckload procurement under shipment uncertainty. Transportation Research Part E: Logistics and Transpor- tation Review, 46(1): 49-60

Qian X, Fang S C, Huang M, An Q, Wang X (2017). Reverse auctions with regret-anticipated bidders. Annals of Operations Research

Qian X, Fang S C, Huang M, Nie T, Wang X (2016). Bidding decisions with nonequilibrium strategic thinking in reverse auctions. Working Paper, 1-28

Rassenti S J, Smith V L, Bulfin R L (1982). A combinatorial auction mechanism for airport time slot allocation. Bell Journal of Economics, 13(2): 402-417

Rekik M, Mellouli S (2012). Reputation-based winner determination problem for combinatorial transportation procurement auctions. Journal of the Operational Research Society, 63(10): 1400-1409

Remli N, Rekik M (2013). A robust winner determination problem for combinatorial transportation auctions under uncertain shipment volumes. Transportation Research Part C: Emerging Technologies, 35: 204-217

Sandholm T (2002). Algorithm for optimal winner determination in combinatorial auctions. Artificial Intelligence, 135(1): 1-54

Singh R K, Benyoucef L (2011). A fuzzy TOPSIS based approach for esourcing. Engineering Applications of Artificial Intelligence, 24(3): 437-448

Srinivasan S, Stallert J, Whinston A B (1998). Portfolio trading and electronic networks. Working Paper, 1-26

Tang C S, Zhang K, Zhou S X (2015). Incentive contracts for managing a project with uncertain completion time. Production and Operations Management, 24(12): 1945-1954

Tunca T I, Wu D J, Zhong F (2014). An empirical analysis of price, quality, and incumbency in procurement auctions. Manufacturing \& Service Operations Management: M \& SOM, 16(3): 346-364

Tversky A, Kahneman D (1992). Advances in prospect theory: Cumulative representation of uncertainty. Journal of Risk and Uncertainty, 5(4): 297-323

Wang X, Wang X, Che H, Li K, Huang M, Gao C (2015). An intelligent economic approach for dynamic resource allocation in cloud services. IEEE Transactions on Cloud Computing, 3(3): 275-289

Wang X, Sun J, Li H, Wu C, Huang M (2013). A reverse auction based allocation mechanism in the cloud computing environment. Applied Mathematics \& Information Sciences, 7(1): 75-84

Zhang B, Yao T, Friesz T L, Sun Y (2015). A tractable two-stage robust winner determination model for truckload service procurement via combinatorial auctions. Transportation Research Part B: Methodological, 78: 16-31

Zhou S X, Tao Z, Zhang N, Cai G G (2016). Procurement with reverse auction and flexible noncompetitive contracts. Decision Sciences, 47 (3): 554-581 